Pacific Journal of Mathematics

CLOSED EXTENSIONS OF THE LAPLACE OPERATOR
DETERMINED BY A GENERAL CLASS OF BOUNDARY

William George BADE AND Robert S. Freeman 


\title{
CLOSED EXTENSIONS OF THE LAPLACE OPERATOR DETERMINED BY A GENERAL CLASS OF BOUNDARY CONDITIONS
}

\author{
W. G. BADE AND R. S. Freeman
}

1. Introduction. This paper is concerned with the spectral theory of closed operators in Hilbert space determined by the Laplace operator and certain general boundary conditions. The method is that of J. W. Calkin $[5,6,7,8]$. In this series of papers Calkin developed a theory of abstract symmetric boundary conditions in Hilbert space, and indicated how these general results might be applied to the Laplace operator and slightly more general operators on certain regions in the plane conformally equivalent to the unit circle. The boundary conditions there are of the type $\partial u / \partial n=L u$, where $L$ is an arbitrary, bounded, self-adjoint operator in $L_{2}(\partial G)$. The potential theoretic details necessary to apply the general results were given in Calkin's thesis, but not published elsewhere. They were subsequently also obtained by J. W. Smith [21] (for the case of the unit circle), who studied cases where the operator $L$ was unbounded. R. S. Freeman [12] extended Calkin's results to a general class of plane domains and obtained a method for treating unbounded domains in $E^{m}$, $m \geqq 2$, once the results were known for bounded domains. In this paper we treat the case of a bounded domain in $E^{m}$ with $C^{1,1}$ boundary. In addition, we extend the method to cover the case for which the operator $L$ in the boundary condition is not necessarily self-adjoint. The case of unbounded domains is treated in another paper [13].

Following Calkin's method, we show there exists an appropriate linear class of functions $\mathscr{D}_{1}(G) \subseteq L_{2}(G)$ such that the operator $S$ in the Hilbert space $L_{2}(G) \oplus L_{2}(\partial G)$ with domain

$$
\mathscr{D}(S)=\left\{[u, \widetilde{u}] \mid u \in \mathscr{D}_{1}(G)\right\}
$$

and

$$
S[u, \tilde{u}]=\left[-\Delta u, \widetilde{u}_{n}\right], \quad u \in \mathscr{D}_{1}(G),
$$

is self-adjoint. Here $\tilde{u}$ and $\widetilde{u}_{n}$ are the values of $u$ and $\partial u / \partial n$ on $\partial G$. If $L$ is an arbitrary (not necessarily self-adjoint) bounded operator in $L_{2}(\partial G)$, then the operator $T_{L}=-\Delta$ on the domain

$$
\mathscr{D}\left(T_{L}\right)=\left\{u \in \mathscr{D}_{1}(G) \mid \widetilde{u}_{n}=L \widetilde{u}\right\}
$$

is closed, and $T_{L}^{*}$ is equal to $T_{L *}$. It is shown that the spectrum of $T_{L}$ is discrete and is contained in a parabola with horizontal axis and opening to the right. One of our aims is a precise determination of $\mathscr{D}_{1}(G)$. It

Received February 20, 1961. This research was supported in part by the National Science Foundation Grant NSF G-5010. 
consists of the class of functions in the domain of the maximal operator associated with $-\Delta$ such that $u$ and $\partial u / \partial n$ have $L_{2}$ boundary values. In potential theoretic terms it is the set of all sums $p+h$, where $p$ is the potential due to a charge $g$ in $G$ and $h$ is a potential due to a surface charge $f$ on $\partial G$, where $g$ and $f$ are, respectively, square integrable over $G$ and $\partial G$ (Theorem 3.4).

Section 2 contains geometric preliminaries and definitions. Section 3 develops the potential theory necessary to define the $S$ operator in $\S 4$. In $\S 5$ certain bounded perturbations of $S$ lead, via a projection argument, to the properties of the operator $T_{L}$. The possibility that $L$ is not selfadjoint leads to new difficulties not treated by previous authors. It should be remarked that Ohdnoff [20] has independently introduced the $S$ operator for general second-order elliptic operators, utilizing the method of Friedrichs. He does not obtain its domain explicitly.

It follows from general results of Browder [2] and others, that when $\partial G$ is $C^{2}$ and $L$ is multiplication by a smooth function, then $\mathscr{D}\left(T_{L}\right) \subseteq$ $W^{2,2}(G)$. It would be interesting to know whether $\mathscr{D}\left(T_{L}\right) \subseteq W^{2,2}(G)$, whenever $L$ is an operator in $L_{2}(\partial G)$ with the property that $L f$ is at least as smooth as $f$.

2. Preliminaries. Throughout the paper $G$ will be a bounded, connected, open set in $E^{m}$ whose boundary is a $C^{1,1}$ surface. The boundary need not be connected, but will necessarily consist of a finite number of components. Points of $G$ will be denoted by $x, y$, etc., and points of $\partial G$ by $\theta, \phi$. At $\theta \in \partial G, n_{\theta}$ will be the exterior unit normal. We summarize first certain properties of such surfaces that we will need. They may be found in the systematic exposition of Lucas [18]. See also [1].

2.1. THEOREM. If $S$ is a $C^{1,1}$ surface, there exists a positive constant $r_{0}$ called the "minimal radius" of $S$, such that

(i) All segments of length $r_{0}$ centered at points of $S$ and normal to $S$ are mutually disjoint and exhaust a neighborhood of $S$.

(ii) At each point $\theta$ of $S$, spheres (exterior and interior) of radius $r_{0}$ can be found tangent to $S$ at $\theta$ which contain no points of $S$.

(iii) The normals to $S$ satisfy a Lipschitz condition with constant $r_{0}^{-1}$ :

$$
\left|n_{\theta}-n_{\phi}\right| \leqq|\theta-\phi| \mid r_{0}, \quad \theta, \phi, \in S .
$$

(iv) The set

$$
S_{\rho}=\left\{\theta-\rho n_{\theta} \mid \theta \in S\right\}
$$

is a $C^{1,1}$ surface for $\rho<r_{0}$ with minimal radius $r_{\rho}$ satisfying $r_{0}-|\rho| \leqq$ $r_{\rho} \leqq r_{0}+|\rho|$. Such surfaces will be called "parallel surfaces to $S$."

(v) The surface measures on $S$ and $S_{\rho}$ are mutually absolutely 
continuous with uniformly bounded Radon-Nikodym derivatives for $|\rho|<r_{0} / 2$.

Let $\theta \in S$ and $\mathscr{T}$ be the tangent plane to $S$ at $\theta$. Choose Cartesian axes with $t=\left(\xi_{1}, \cdots, \xi_{m-1}\right) \in \mathscr{T}$ and positive $\xi_{m}$ axis along the inner normal to $S$ at $\theta$. Let $\lambda(t),|t|<r_{0}$, be the function representing $S$ locally near $\theta$. We shall need the following facts.

2.2. Lemma. There exist constants $k_{1}, k_{2}$, and $\rho_{0}$ independent of $\theta$ such that

$$
\begin{gathered}
|\lambda(t)| \leqq k_{1}|t|^{2}, \quad|t|<r_{0} / 2 \\
{\left[\sum_{i=1}^{m-1}\left(\partial \lambda / \partial \xi_{i}\right)^{2}\right]^{1 / 2} \leqq k_{2}|t|, \quad|t|<r_{0} / 2} \\
\left|\phi-\rho n_{\phi}-\theta\right|^{2} \geqq \frac{1}{2}\left[|t|^{2}+\rho^{2}\right], \quad \rho<\rho_{0},
\end{gathered}
$$

where $\phi=[t, \lambda(t)],|t|<r_{0} / 2$.

Proof. Statement (a) follows from (ii) above, while (b) follows from (iii). To prove (c) we note

$$
\begin{aligned}
\left|\phi-\rho n_{\phi}-\theta\right|^{2} & =\sum_{i=1}^{m-1}\left|\xi_{i}-\rho \Omega(t)^{-1} \partial \lambda / \partial \xi_{i}\right|^{2}+\left|\lambda(t)+\rho \Omega(t)^{-1}\right|^{2} \\
= & \rho^{2}+|t|^{2}+|\lambda(t)|^{2}+2 \rho \Omega(t)^{-1}\left[\lambda(t)-\sum_{i=1}^{m-1} \xi_{i} \partial \lambda / \partial \xi_{i}\right],
\end{aligned}
$$

where

$$
\Omega(t)=\left[1+\sum_{i=1}^{m-1}\left(\partial \lambda / \partial \xi_{i}\right)^{2}\right]^{1 / 2}
$$

Thus we have

$$
\left|\phi-\rho n_{\phi}-\theta\right|^{2} \geqq \rho^{2}+|t|^{2}-2 \rho\left(k_{1}+k_{2}\right)|t|^{2},
$$

which yields the desired result with $\rho_{0}=4^{-1}\left(k_{1}+k_{2}\right)^{-1}$.

Next we shall need certain classes of functions on $G$. In the notation of Browder [3], $W^{n, p}(G)$ is the class of all functions in $L^{p}(G), 1 \leqq p<\infty$, all of whose distribution derivatives of order $\leqq n$ are functions of $L^{p}(G)$. It is a Banach space under the norm which is the sum of the $L_{p}$ norms of all derivatives of order $\leqq n$. We shall be concerned only with the cases $n=1$ and 2 .

Let $u$ be a function defined on the intersection of $G$ with a neighborhood of $\partial G$. Let $\rho<r_{0}$ and $u_{\rho}(\theta)=u\left(\theta-\rho n_{\theta}\right), \theta \in \partial G$. Thus $u_{\rho}$ is the restriction of $u$ to the parallel surface at a distance $\rho$ along the normal. If $u_{\rho} \in L_{p}(\partial G)$ and $\widetilde{u}=\lim _{\rho \rightarrow 0} u_{\rho}$ exists in $L_{p}(\partial G)$, we say $u$ has the $L_{p}$ boundary value $\tilde{u}$. It is known that every function in $W^{1, p}(G)$ has an $L^{p}$ - boundary value. (See for example [19].) 
The following class of functions will be of basic importance throughout the paper.

2.3. Definition. We denote by $\mathscr{D}_{1}(G)$ the class of functions $u \in L_{2}(G)$ such that

(i) $u$ belongs to $W^{2,2}\left(G_{1}\right)$ for each open set $G_{1}$ whose closure lies in $G$,

(ii) $\Delta u \in L_{2}(G)$,

(iii) $u$ and $\partial u / \partial n$ have $L_{2}$ boundary values $\tilde{u}$ and $\tilde{u}_{n}$ on $\partial G$.

Clearly, $W^{2,2}(G) \subseteq \mathscr{D}_{1}(G) \subseteq W^{1,2}(G)$. The inclusions are proper (as one sees, for example, by considering the case of harmonic functions in the unit circle). Conditions (i) and (ii) are the conditions that $u$ should lie in the domain of the "maximal operator" corresponding to the Laplacian on $G$-that is, the adjoint of the "minimal operator" which is the closure of the Laplacian on $C^{\infty}$ functions with compact support.

It follows easily from Theorem 2.1 (v) that Green's identities hold for functions in $\mathscr{D}_{1}(G)$ :

$$
\int_{G}(-\Delta u) \bar{v} d x=\int_{\theta} \sum_{i=1}^{m} \frac{\partial u}{\partial x_{i}} \frac{\partial \bar{v}}{\partial x_{i}} d x-\int_{\partial G} \widetilde{u}_{n} \overline{\bar{v}} d \theta, \quad u, v \in \mathscr{D}_{1}(G)
$$

II

$$
\int_{G}(-\Delta u) \bar{v} d x-\int_{\theta} u(\overline{(-\Delta v}) d x=\int_{\partial G}\left(\tilde{u} \overline{\widetilde{v}}_{n}-\widetilde{u}_{n} \overline{\widetilde{v}}\right) d \theta, \quad u, v \in \mathscr{D}_{1}(G) .
$$

3. Potential theory. In this section we prove that the inhomogeneous Neumann problem

$$
-\Delta u=0, \quad \partial u / \partial n=k \text { on } \partial G
$$

has a solution in $\mathscr{D}_{1}(G)$ whenever $k \in L_{2}(\partial G)$ and $\int_{\partial G} k(\theta) d \theta=0$. This fact will be needed in later sections. The solution $u$ will be a single-layer potential

$$
u(x)=\frac{1}{m-2} \int_{\partial \theta} \frac{f(\theta) d \theta}{|x-\theta|^{m-2}}, x \in G, f \in L_{2}(\partial G), m \geqq 3,
$$

or

$$
u(x)=-\int_{\partial \theta} f(\theta) \log |x-\theta| d \theta, x \in G, f \in L_{2}(\partial G), m=2 .
$$

The argument that for such potentials both $u$ and $\partial u / \partial n$ have boundary values works equally well in $L_{p}, 1<p<\infty$, and we present it in that generality for convenience of reference for a later paper.

If $u$ is a single-layer potential with charge $f$ in $L_{p}(\partial G)$, then

$$
\frac{\partial u}{\partial n}\left(\phi-\rho n_{\phi}\right)=\int_{\partial G} N_{\rho}(\phi, \theta) f(\theta) d \theta,
$$


where, writing $\phi-\rho n_{\phi}=\phi_{\rho}$, we have

$$
N_{\rho}(\phi, \theta)=-\frac{\cos \left(\phi_{\rho}-\theta, n_{\phi}\right)}{\left|\phi_{\rho}-\theta\right|^{m-1}}, \quad m \geqq 2 .
$$

Let $T_{\rho}$ be the bounded linear mapping of $L_{p}(\partial G)$ into itself which carries $f$ into the function $\left(T_{\rho} f\right)(\phi)=(\partial u / \partial n)\left(\phi_{\rho}\right), \phi \in \partial G$. It is well known that when $f$ has a continuous derivative

$$
\lim _{\rho \rightarrow 0}\left(T_{\rho} f\right)(\phi)=\frac{1}{2} \omega_{m-1} f(\phi)-\int_{\partial \theta} \frac{\cos \left(\phi-\theta, n_{\phi}\right)}{|\phi-\theta|^{m-1}} f(\theta) d \theta, \phi \in \partial G,
$$

uniformly on $\partial G$, where $\omega_{m-1}$ is the volume of the unit sphere in $E^{m-1}$. Thus to prove that $\partial u / \partial n$ has an $L_{p}$ boundary value, it suffices to prove that the maps $T_{\rho}$ are uniformly bounded in $L_{p}(\partial G)$. Then the integral in formula $(*)$ will determine a bounded linear map, and $(*)$ will hold for all $f$ in $L_{p}(\partial G)$. To prove the maps $T_{\rho}$ are uniformly bounded, it suffices (cf. [9], page 518) to prove the existence of constants $M$ and $\rho_{0}$ such that

$$
\begin{aligned}
& \sup _{\theta \in \partial G} \int_{\partial G}\left|N_{\rho}(\phi, \theta)\right| d \phi \leqq M, \rho<\rho_{0} . \\
& \sup _{\phi \in \partial G} \int_{\partial G}\left|N_{\rho}(\phi, \theta)\right| d \theta \leqq M, \rho<\rho_{0} .
\end{aligned}
$$

Then $\left\|T_{\rho}\right\| \leqq M, \rho<\rho_{0}$.

We first prove (i). Let $\theta$ be any fixed point of $\partial G$ and $\mathscr{T}$ be the tangent plane to $\partial G$ at $\theta$. Choose Cartesian axes for $E^{m}$ with origin at $\theta, t=\left(\xi_{1}, \cdots, \xi_{m-1}\right) \in \mathscr{T}$ and positive $\xi_{m}$ axis along the inner normal at $\theta$. Let $\xi_{m}=\lambda(t),|t|<r_{0}$, be the $C^{1,1}$ function representing $\partial G$ locally near $\theta$. Then we have

$$
\begin{aligned}
\left|\phi_{\rho}-\theta\right| \cos \left(\phi_{\rho}-\theta, n_{\phi}\right)=\Omega(t)^{-2}\left[\Omega(t)\left(\sum_{i=1}^{m-1} \xi_{i} \frac{\partial \lambda}{\partial \xi_{i}}\right)\right. & -\rho \sum_{i=1}^{m-1}\left|\frac{\partial \lambda}{\partial \xi_{i}}\right|^{2} \\
& -\lambda(t) \Omega(t)-\rho],
\end{aligned}
$$

where

$$
\Omega(t)=\left[1+\sum_{i=1}^{m-1}\left(\frac{\partial \lambda}{\partial \xi_{i}}\right)^{2}\right]^{1 / 2}
$$

and $t=P \phi, P$ being the perpendicular projection of $E^{m}$ onto $\mathscr{T}$. Now by Lemma 2.2 (c) we have

$$
\left|\phi_{\rho}-\theta\right|^{2} \geqq \frac{1}{2}\left[|t|^{2}+\rho^{2}\right], \phi \in C\left(\theta, \frac{1}{2} r_{0}\right)=\partial G \cap\left\{x|| x-\theta \mid<\frac{1}{2} r_{0}\right\},
$$


for all small $\rho$. Combining this fact with 2.2 (a) and (b), we see

$$
\left|N_{\rho}(\phi, \theta)\right| \leqq \frac{C_{1}}{|t|^{m-2}}+\frac{C_{2} \rho}{\left[|t|^{2}+\rho^{2}\right]^{m / 2}}, \quad t=P \phi, \phi \in C\left(\theta, \frac{1}{2} r_{0}\right),
$$

for all $\rho$ less than a suitable $\rho_{0}$, where $C_{1}$ and $C_{2}$ are constants independent of $\rho$ and $\theta$. The integral (with respect to $t$ ) of the right side is bounded independent of $\rho$ over $|t|<r_{0} / 2$; so therefore

$$
\sup _{\theta \in \partial G} \int_{O\left(\theta,(1 / 2) r_{0^{\prime}}\right.}\left|N_{\rho}(\phi, \theta)\right| d \phi<\infty, \rho<\rho_{0} .
$$

Since

$$
\left|\phi_{\rho}-\theta\right| \geqq|\phi-\theta|-\rho \geqq r_{0} / 4
$$

if $\rho<r_{0} / 4$ and $|\phi-\theta|>r_{0} / 4$, it follows that $\left|N_{\rho}(\phi, \theta)\right|$ is uniformly bounded independent of $\theta$ if $|\phi-\theta|>r_{0} / 2$. Thus (i) follows. The proof of (ii) is simpler. If we now take the tangent plane at $\phi$ instead of $\theta$, $\left|\phi_{\rho}-\theta\right| \cos \left(\phi_{\rho}-\theta, n_{\phi}\right)=\lambda(t)-\rho, t=P \theta$, and similar estimates apply. Thus we have

3.1. Theorem. Let $1<p<\infty$, and $u$ be the single-layer potential in $G$ with charge $f$, where $f \in L_{p}(\partial G)$. Then $u$ and $\partial u / \partial n$ have $L_{p}$ boundary values $\tilde{u}$ and $\widetilde{u}_{n}$ given by the formulas

$$
\begin{aligned}
\tilde{u}(\phi) & =-\int_{\partial \theta} f(\theta) \log |\phi-\theta| d \theta, \quad m=2 \\
& =\frac{1}{m-2} \int_{\partial \theta} \frac{f(\theta) d \theta}{|\phi-\theta|^{m-2}}, \quad m \geqq 3
\end{aligned}
$$

and

$$
\tilde{u}_{n}(\phi)=\frac{1}{2} \omega_{m-1} f(\phi)-\int_{\partial G} \frac{\cos \left(\phi-\theta, n_{\phi}\right)}{|\phi-\theta|^{m-1}} f(\theta) d \theta, \phi \in \partial G
$$

The maps $B: f \rightarrow \widetilde{u}$ and $T: f \rightarrow \widetilde{u}_{n}$ are bounded linear maps in $L_{p}(\partial G)$.

The fact that $\partial u / \partial n$ has a boundary value implies the same for $u$, as one sees by an elementary estimate from the formula

$$
u\left(\theta-\rho_{1} n_{\theta}\right)-u\left(\theta-\rho_{2} n_{\theta}\right)=-\int_{\rho_{2}}^{\rho_{1}} \frac{\partial u}{\partial n}\left(\theta-\alpha n_{\theta}\right) d \alpha .
$$

The formula for $\tilde{u}$ follows from the fact it is valid for $C^{1}$ functions.

3.2. CoRollary. If $u$ is a single-layer potential with charge in $L_{2}(\partial G)$, then $u \in \mathscr{D}_{1}(G)$. 
3.3. Theorem. Let $k \in L_{p}(\partial G), 1<p<\infty$, and suppose $\int_{\partial \theta} k(\theta) d \theta=$ 0 . Then there exists a single-layer potential with charge in $L_{p}(\partial G)$ such that $\widetilde{u}_{n}=k$.

Proof. It suffices by Theorem 2.1, to find a function $f$ in $L_{p}(\partial G)$ which satisfies the integral equation

$$
f(\phi)-\int_{\partial \theta} K(\phi, \theta) f(\theta) d \theta=\hat{k}(\phi),
$$

where $\hat{k}=2 \omega_{m-1}^{-1} k$ and

$$
K(\phi, \theta)=\frac{2}{\omega_{m-1}} \frac{\cos \left(\phi-\theta, n_{\phi}\right)}{|\phi-\theta|^{m-1}} .
$$

The Fredholm theory is applicable to this equation since an appropriate iterate of the kernel $K$ is bounded (cf. the discussion in [22], Chapter $\mathrm{XI}$ and [11], p. 542). Thus equation $\left(^{*}\right)$ has a solution $f$ if and only if $\int_{\partial \theta} k(\theta) \overline{l(\theta)} d \theta=0$ for all solutions $l$ in $L_{q}(\partial G), p^{-1}+q^{-1}=1$, of the adjoint homogeneous equation

$$
l(\theta)=\int_{\partial G} K(\phi, \theta) l(\phi) d \phi .
$$

It is well known that the constant functions are the only continuous solutions of this equation. However, any solution $l$ also satisfies the equation obtained by replacing $K$ by any of its iterates. Since an appropriate iterate is bounded, $l$ is continuous and hence constant. Thus equation $\left(^{*}\right)$ has a solution if and only if $\int_{\partial G} k(\theta) d \theta=0$.

Returning now to the case $p=2$, we seek a potential theoretic characterization of $\mathscr{D}_{1}(G)$. If $g \in L_{2}(G)$, the function

$$
\begin{aligned}
p(x) & =\int_{\theta} \frac{g(y)}{|x-y|^{m-2}} d y, \quad m \geqq 3 \\
& =-\int_{\theta} g(y) \log |x-y| d y, \quad m=2,
\end{aligned}
$$

will be called the space potential with charge $g$. Since $G$ is a bounded. domain, $p$ belongs to $W^{2,2}(G)$, and $-\Delta p=m \omega_{m} g$ almost everywhere in $G$. These facts may be derived from [4], pp. 130, 134. There the point-wise existence of the first two derivatives is established almost everywhere. Since they are given by formulas that define bounded maps in $L_{2}(G)$, it follows that $u \in W^{2,2}(G)$.

3.4. Theorem. A function $u$ belongs to $\mathscr{D}_{1}(G)$ if and only if $u=$ 
$p+h$, where $p$ is a space potential due to a charge in $L_{2}(G)$, and $h$ is a single-layer potential with charge in $L_{2}(\partial G)$.

Proof. If $u$ has this form, then, clearly, $u \in \mathscr{D}_{1}(G)$. To prove the converse, let $u$ be given in $\mathscr{D}_{1}(G)$ and let $p$ be the space potential whose charge is $-\left(m \omega_{m}\right)^{-1} \Delta u$. Then $\Delta p=\Delta u$, and therefore $v=u-p$ is harmonic and belongs to $\mathscr{D}_{1}(G)$. By Green's second identity, $\int_{\partial G} \widetilde{v}(\theta) d \theta=0$; thus by Theorem 3.3 there exists a single layer potential $w$ such that $\widetilde{w}_{n}=\widetilde{v}_{n}$. But then Green's first identity implies $w-v$ is constant. However, the function 1 in $G$ is a single layer potential. Its charge is the (normalized) solution of the homogeneous equation

$$
f(\phi)=\int_{\partial G} K(\phi, \theta) f(\theta) d \theta .
$$

It follows from the Riesz theory that the set of solutions is one dimensional (since this was the case for the adjoint equation).

We next consider the inhomogeneous Neumann problem.

3.5. Theorem. Let $g \in L_{2}(G), l \in L_{2}(\partial G)$. The equations

$$
-\Delta u=g, \quad \tilde{u}_{n}=l
$$

have a solution $u$ in $\mathscr{D}_{1}(G)$ if and only if

$$
\int_{G} g(x) d x+\int_{\partial G} l(\theta) d \theta=0 .
$$

Proof. Suppose condition $\left({ }^{*}\right)$ holds. Let $p$ be the space potential with charge $\left(m \omega_{m}\right)^{-1} g$. Then $p \in W^{2,2}(G)$ and $-\Delta p=g$. By Green's identity.

$$
\int_{\partial \theta} g(x) d x+\int_{\partial \theta} \widetilde{p}_{n}(\theta) d \theta=0 .
$$

Thus $\int_{\partial \theta}\left[\widetilde{p}_{n}(\theta)-l(\theta)\right] d \theta=0$, and therefore by Theorem 3.3 there exists a harmonic function $v$ in $\mathscr{D}_{1}(G)$ such that $\widetilde{v}_{n}=\widetilde{p}_{n}-l$. The function $u=p-v$ solves the problem. Conversely, if $u$ is a solution, formula (*) results from applying Green's identity to $u$ and 1 .

3.6. Theorem. Let $g \in L_{2}(G)$. The equations

$$
-\Delta u=g, \quad \tilde{u}_{n}=0
$$

have a solution $u$ in $\mathscr{D}_{1}(G)$ if and only if $\int_{\theta} g d x=0$. The operator $T_{N}$ in $L_{2}(G)$ with domain $\mathscr{D}\left(T_{N}\right)=\left\{u \in \mathscr{D}_{1}(G) \mid \tilde{u}_{n}=0\right\}$ and $T_{N} u=-\Delta u$ is self-adjoint. Moreover $\mathscr{R}\left(T_{N}\right)=\mathscr{N}\left(T_{N}\right)^{\perp}$, the null manifold consisting of the constant functions. 
Proof. The first statement follows from the previous theorem. To prove the second, we recall that the method of Friedrichs [14] yields a selfadjoint operator $F_{N}$ in $L_{2}(G)$ corresponding to $-\Delta$ and the boundary condition $\partial u / \partial n=0$ in $\partial G$. Explicitly, consider the closed semi-bounded form

$$
D(u, v)=\int_{\theta} \sum_{i=1}^{m} \frac{\partial u}{\partial x_{i}} \frac{\partial \bar{v}}{\partial x_{i}} d x
$$

on the dense subspace $W^{1,2}(G) \subseteq L_{2}(G)$. Then $u \in \mathscr{D}\left(F_{N}\right)$ if and only if there exists an element $g \in L_{2}(G)$ such that

$$
D(u, v)=(g, v), \quad \text { all } v \in W^{1,2}(G),
$$

and $F_{N} u=g$ for $u \in \mathscr{D}\left(F_{N}\right)$. The range of $F_{N}$ is closed and is the orthogonal complement of its null manifold, which clearly consists of the constant functions. Thus $\mathscr{N}\left(T_{N}\right)=\mathscr{N}\left(F_{N}\right), \mathscr{R}\left(T_{N}\right)=\mathscr{R}\left(F_{N}\right)$ and $T_{N} \leqq$ $F_{N}$. However, if $u \in \mathscr{D}\left(F_{N}\right)$, then $F_{N} u=T_{N} v$ for some $v \in \mathscr{D}\left(T_{N}\right)$; therefore $F_{N}(u-v)=0$. Thus $u=v+$ constant $\in \mathscr{D}\left(T_{N}\right)$, showing $F_{N}=T_{N}$.

REMark. It is known [2], that when $\partial G$ is of class $C^{2}$, then $\mathscr{D}\left(F_{N}\right) \cong W^{2,2}(G)$.

4. The $S$ operator. In this section, following Calkin [6], page 435, we introduce the operator $S$ in $L_{2}(G) \oplus L_{2}(\partial G)$. In the next section, certain bounded perturbations of $S$ lead, via a projection argument, to closed linear operators in $L_{2}(G)$ corresponding to restricting $-\Delta$ by boundary conditions.

4.1. Definition. In the Hilbert space, $L_{2}(G) \oplus L_{2}(\partial G)$ define the operator $S$ with domain

$$
\mathscr{D}(S)=\left\{[u, \tilde{u}] \mid u \in \mathscr{D}_{1}(G)\right\}
$$

and

$$
S[u, \widetilde{u}]=\left[-\Delta u, \widetilde{u}_{n}\right], \quad[u, \widetilde{u}] \in \mathscr{D}(S) .
$$

As we shall see, the theory of the $S$ operator is intimately connected to the inhomogeneous Neumann problem of Theorem 3.5.

4.2. THEOREM. The operator $S$ is densely defined, closed, selfadjoint, and has compact resolvent.

Proof. To see that $\mathscr{D}(S)$ is dense in $\mathscr{C}=L_{2}(G) \oplus L_{2}(\partial G)$, suppose

$$
([u, \widetilde{u}],[v, l])=(u, v)+(\widetilde{u}, l)=0
$$


for all $u \in \mathscr{D}_{1}(G)$. Then (taking $u=1$ ) we see

$$
\int_{\theta} v d x+\int_{\partial G} l d \theta=0
$$

consequently there exists $g \in \mathscr{D}_{1}(G)$ such that $-\Delta g=v, \widetilde{g}_{n}=l$. Thus, using Green's formula, we have

$$
(-\Delta u, g)+\left(\widetilde{u}_{n}, \widetilde{g}\right)=(u, v)+(\widetilde{u}, l)=0, \quad u \in \mathscr{D}_{1}(G) .
$$

If we consider only those $u$ such that $\tilde{u}_{n}=0$, we can show that $g \in \mathscr{R}\left(T_{n}\right)^{\perp}$. It follows from Theorem 3.6 that $g$ is constant. Thus $v$ and $l$ are zero.

Since $\mathscr{D}(S)$ is dense, $S^{*}$ exists, and Green's formula shows $S \subseteq S^{*}$. Suppose now that $[w, j] \in \mathscr{D}\left(S^{*}\right)$. There exists an element $[v, l]$ in $\mathscr{H}$ such that

$$
(S[u, \widetilde{u}],[w, j])=\left([u, \widetilde{u}],{ }_{,}^{5}[v, l]\right), \quad u \in \mathscr{D}_{1}(G)
$$

i.e.,

$$
\left.(-\Delta u, w)+\left(\widetilde{u}_{n}, j\right)=(u, v)\right]+\left(\widetilde{u}_{a}^{\prime} l\right), \quad u \in \mathscr{D}_{1}(G) .
$$

Thus (taking $u=1$ ) we have

$$
\int_{\theta} v d x+\int_{\partial \theta} l d \theta=0 .
$$

Consequently, by Theorem 3.5 we can find $z \in \mathscr{D}_{1}(G)$ such that $-4 z_{d i d}^{\prime}=$ $v, \widetilde{z}_{n}=l$. By Green, again, we have

$$
\begin{aligned}
(-\Delta u, z)+\left(\widetilde{u}_{n}, \widetilde{z}\right) & =(u,-\Delta z)+\left(\widetilde{u}, \widetilde{z}_{n}\right) \\
& =(u, v)+(\widetilde{u}, l), \quad u \in \mathscr{D}_{1}(G),
\end{aligned}
$$

and so

$$
(-\Delta u, z-w)+\left(\widetilde{u}_{n}, \widetilde{z}-j\right)=0, u \in \mathscr{D}_{1}(G) .
$$

Restricting attention to those $u$ with $\widetilde{u}_{n}=0$ shows $z-w$ is constant. Thus $w \in \mathscr{D}_{1}(G),-\Delta w=v$, and $\widetilde{w}_{n}=l$. Putting these facts in $\left(^{* *}\right)$ and applying Green again, we obtain

$$
\left(\widetilde{u}_{n}, j-\widetilde{w}\right)=0, \quad u \in \mathscr{D}_{1}(G) .
$$

One sees easily from Theorem 3.5 that there are functions $u$ in $\mathscr{D}_{1}(G)$ for which $\widetilde{u}_{n}$ takes arbitrary values in $L_{2}(\partial G)$. Thus $\widetilde{w}=j$. Hence $[w, j]=[w, \widetilde{w}] \in \mathscr{D}(S)$ and $[v, l]=S[w, \widetilde{w}]$, proving $S$ is self-adjoint.

If $\mathscr{I}(\lambda) \neq 0$ and $(\lambda I-S)\left[v^{k}, \widetilde{v}^{k}\right], k=1,2, \cdots$, is a bounded sequence in $\mathscr{H}$, the sequence $\left[v^{k}, \widetilde{v}^{k}\right]$ is bounded, since $(\lambda I-S)^{-1}$ is bounded. Thus

$$
D\left(v^{k}, v^{k}\right)=\left(-\Delta v^{k}, v^{k}\right)+\left(\widetilde{v}_{n}^{k}, \widetilde{v}\right)
$$


is bounded and the $v^{k}$ are bounded in $W^{1,2}(G)$. Since the maps $v \rightarrow v$ and $v \rightarrow \widetilde{v}$ of $W^{1,2}(G)$ into $L_{2}(G)$ and $L_{2}(\partial G)$ are compact (cf. [19], p. 208), we can extract a sub-sequence of the $\left[v^{k}, \widetilde{v}^{k}\right]$ converging in $\mathscr{H}$, showing $(\lambda I-S)^{-1}$ is compact.

4.3. CoRollary. The range of $S$ is closed, $\mathscr{R}(S)=\mathscr{N}(S)^{+}$, and $\mathscr{N}(S)$ consists of the constants $[\alpha, \alpha]$ in $L_{2}(G) \oplus L_{2}(\partial G)$.

5. Boundary conditions for -4 . We now proceed to the main results of the paper.

5.1. Definition. Let $L$ be an arbitrary bounded operator in $L_{2}(\partial G)$. Define the operator $T_{L}$ in $L_{2}(G)$ with domain

$$
\begin{aligned}
\mathscr{D}\left(T_{L}\right) & =\left\{u \mid u \in \mathscr{D}_{1}(G), \tilde{u}_{n}-L \tilde{u}=0\right\}, \\
T_{L} u & =-\Delta u, u \in \mathscr{D}\left(T_{L}\right) .
\end{aligned}
$$

It will be shown that $T_{L}$ is a closed linear operator with compact resolvent, and $\left(T_{L}\right)^{*}=T_{L *}$. Thus $T_{L}$ is self-adjoint if $L=L^{*}$. These results will be proved by perturbation of the operator $S$ and a projection argument.

5.2. Definition. Let $L$ be a bounded operator in $L_{2}(\partial G)$, and $\lambda$ a complex number. The operators $\hat{L}$ and $P(\lambda)$ are defined in $L_{2}(G) \bigoplus L_{2}(\partial G)$ by the formulas

$$
\begin{aligned}
\hat{L}([f, k]) & =[0, L k], & & {[f, k] \in L_{2}(G) \oplus L_{2}(\partial G) . } \\
P(\lambda)([f, k]) & =[\lambda f, 0], & & {[f, k] \in L_{2}(G) \bigoplus L_{2}(\partial G) . }
\end{aligned}
$$

5.3. Lemma. $\|\hat{L}\|=\|L\|,\|P(\lambda)\|=|\lambda|$, and $(\hat{L})^{*}=\left(L^{*}\right)^{\wedge}, P(\lambda)^{*}=$ $P(\bar{\lambda})$. Note that the operator $S-\hat{L}-P(\lambda)$ carries $[u, \tilde{u}]$ into $[-\Delta u-\lambda u$, $\left.\tilde{u}_{n}-L \tilde{u}\right]$.

5.4. Lemma. The operator $S-\hat{L}-P(\lambda)$ is closed, densely defined in $L_{2}(G) \oplus L_{2}(\partial G)$, and has compact resolvent. Thus, $\mathscr{R}(S-\hat{L}-P(\lambda))=$ $\mathscr{N}\left(S-\hat{L}^{*}-P(\bar{\lambda})\right)^{+}$.

Proof. It is well known that a bounded perturbation of an unbounded self-adjoint operator with compact resolvent is a closed operator with compact resolvent (cf. [6], p. 435). The last statemant then follows from the Riesz theory.

Now consider the case $\lambda=0$. The general element of the graph $\mathscr{G}(S-\hat{L})$ in $L_{2}(G) \oplus L_{2}(\partial G) \oplus L_{2}(G) \oplus L_{2}(\partial G)$ has the form

$$
\left[u, \tilde{u},-\Delta u, \widetilde{u}_{n}-L \tilde{u}\right], \quad u \in \mathscr{D}_{1}(G) \text {. }
$$


Moreover, $\mathscr{C}(S-\hat{L})$ is a closed linear manifold. We denote by $\mathscr{K}$ the submanifold consisting of all those elements of $\mathscr{G}(S-\hat{L})$ for which $\tilde{u}_{n}-L \tilde{u}=0$. Clearly, $\mathscr{K}$ is also closed. The map $J: \mathscr{K} \rightarrow L_{2}(G) \oplus L_{2}(G)$ is defined by the equation

$$
J[u, \tilde{u}-\Delta u, 0]=[u,-\Delta u] .
$$

The following facts are immediate consequences of this definition.

5.5. Lemma. The mapping $J$ is a one-to-one linear mapping of $K$ onto the graph $\mathscr{C}\left(T_{L}\right)$ of $T_{L}$ in $L_{2}(G) \oplus L_{2}(G)$, and $\|J\| \leqq 1$.

5.6. Lemma. The operator $T_{L}$ is a closed linear operator in $L_{2}(G)$ with dense domain.

Proof. Since $C_{0}^{\infty}(G) \subseteq \mathscr{D}\left(T_{L}\right)$, the domain is dense. To prove $T_{L}$ is closed it suffices to prove that $J$ has a closed range in $L_{2}(G) \oplus L_{2}(G)$. For this it is enough to show that $J$ maps bounded closed sets onto closed sets (cf. [9], page 489).

Let $B$ be a bounded closed set in $\mathscr{K}$ and $\left[u^{k}, \widetilde{u}^{k},-\Delta u^{k}, 0\right]$, $k=1,2, \cdots$, be a sequence in $B$ such that $\left[u^{k},-\Delta u^{k}\right]$ converges in $L_{2}(G) \oplus L_{2}(G)$. By Green's identity we have

$$
\begin{aligned}
D\left(u^{k}, u^{k}\right) & =\left(-\Delta u^{k}, u^{k}\right)-\left(\widetilde{u}_{n}^{k}, \widetilde{u}^{k}\right) \\
& =\left(-\Delta u^{k}, u^{k}\right)-\left(L \widetilde{u}^{k}, \widetilde{u}^{k}\right) \\
& \leqq(1+\|L\|) M^{2},
\end{aligned}
$$

where $M$ is a bound for $B$. It follows that $\left\{u^{k}\right\}$ is bounded in $W^{1,2}(G)$. A sub-sequence (which we may take to be $\left\{u^{k}\right\}$ ) converges weakly in $W^{1,2}(G)$, and therefore $\left\{\widetilde{u}^{k}\right\}$ converges strongly in norm (cf. [19], page 208). Thus $\left[u^{k}, \widetilde{u}^{k},-\Delta u^{k}, 0\right]$ converges, and so $J(B)$ is closed.

5.7. Corollary. There exists a constant $K_{L}$ such that

$$
\|\tilde{u}\|^{2} \leqq K_{L}\left(\|u\|^{2}+\|\Delta u\|^{2}\right)^{1 / 2}, \quad u \in \mathscr{D}\left(T_{L}\right) .
$$

Proof. Since $J$ is one-to-one and maps onto a closed manifold, its inverse is continuous.

It follows from Green's formula that $T_{L *} \subseteq T_{L}{ }^{*}$. Our next objective is to prove the two operators are equal. This will be done by consideration of ranges and null manifolds.

5.8. Lemma. Let $\lambda$ be any complex number. Then we have

$$
\begin{aligned}
& \mathscr{R}\left(T_{L}-\lambda I\right)=\left\{g \in L_{2}(G) \mid[g, 0] \in \mathscr{R}(S-\hat{L}-P(\lambda))\right\}, \\
& \mathscr{N}\left(T_{L}-\lambda I\right)=\left\{u \in \mathscr{D}_{1}(G) \mid[u, \tilde{u}] \in \mathscr{N}^{\prime}(S-\hat{L}-P(\lambda))\right\} .
\end{aligned}
$$


Proof. If $[g, 0] \in \mathscr{R}(S-\hat{L}-P(\lambda))$, there exists $u \in \mathscr{D}_{1}(G)$ such that $-\Delta u-\lambda u=g, \widetilde{u}_{n}-L \widetilde{u}=0$. Thus $g \in \mathscr{R}\left(T_{L}-\lambda I\right)$. Conversely, it is clear that if $g \in \mathscr{R}\left(T_{L}-\lambda I\right)$, then $[g, 0] \in \mathscr{R}(S-\hat{L}-P(\lambda))$. The proof of $(b)$ is equally direct.

5.9. Lemma. For each complex $\lambda$, we have

$$
\mathscr{R}\left(T_{L}-\lambda I\right)=\mathscr{N}\left(T_{L *}-\bar{\lambda} I\right)^{+} ;
$$

therefore, in particular, $\mathscr{R}\left(T_{L}-\lambda I\right)$ is closed.

Proof. We know $g \in \mathscr{R}\left(T_{L}-\lambda I\right)$ if and only if $[g, 0] \in \mathscr{R}(S-\hat{L}-P(\lambda))$. By Lemma 5.4, however, the latter manifold is $\mathscr{N}\left(S-\hat{L}^{*}-P(\bar{\lambda})\right)^{\perp}$, and clearly

$$
([g, 0],[u, \tilde{u}])=0,[u, \widetilde{u}] \in \mathscr{N}\left(S-\hat{L}^{*}-P(\bar{\lambda})\right)
$$

if and only if

$$
(g, u)=0, u \in \mathscr{N}\left(T_{L *}-\bar{\lambda} I\right)
$$

5.10. Lemma.

$$
\left(T_{L}\right)^{*}=T_{L *}
$$

Proof. Taking $\lambda=0$ in the last lemma, we have $\mathscr{R}\left(T_{L}\right)=\mathscr{N}\left(T_{L *}\right)^{\perp}$. But since $\mathscr{R}\left(T_{L}\right)$ is closed, $\mathscr{R}\left(T_{L}\right)=\mathscr{N}\left(\left(T_{L}\right)^{*}\right)^{\perp}$. Since $T_{L}$ and $T_{L *}$ are closed, so are their null manifolds, and

$$
\mathscr{N}\left(T_{L}^{*}\right)=\mathscr{N}\left(T_{L *}\right) \text {. }
$$

Because $\mathscr{R}\left(T_{L}\right)$ is closed, so is $\mathscr{R}\left(T_{L}{ }^{*}\right)$ (cf. [15]). Now $T_{L}=T_{L}{ }^{* *}$, showing

$$
\mathscr{R}\left(T_{L}^{*}\right)=\mathscr{N}\left(T_{L}^{* *}\right)^{+}=\mathscr{N}\left(T_{L}\right)^{+}=\mathscr{R}\left(T_{L *}\right) .
$$

From these facts we can show that $T_{L}{ }^{*} \subseteq T_{L *}$, as follows: If $u \in \mathscr{D}\left(T_{L}{ }^{*}\right)$, we can find $y$ in $\mathscr{D}\left(T_{L *}\right)$ by (ii) such that $T_{L *} y=T_{L}{ }^{*} u$. Because $T_{L *} \subseteq T_{L}{ }^{*}, T_{L}{ }^{*}(y-u)=0$, and therefore, by (i), $y-u \in \mathscr{D}\left(T_{L *}\right)$. Thus $u \in \mathscr{D}\left(T_{L *}\right)$, and the required inclusion follows directly,

It remains to prove $T_{L}$ has compact resolvent. As we shall see, this is easy to prove if it can be shown that the resolvent set $\rho\left(T_{L}\right)$ of $T_{L}$ is not empty. Again the problem can be thrown onto $S$.

5.11. Lemma. A complex number $\lambda$ is in $\rho\left(T_{L}\right)$ if and only if 0 is in $\rho(S-\hat{L}-P(\lambda))$.

Proof. By Lemma 5.8 (b), $\lambda$ is an eigenvalue of $T_{L}$ if and only if 0 is an eigenvalue of $S-\hat{L}-P(\lambda)$. If 0 is in the spectrum of 
$S-\hat{L}-P(\lambda)$, it must be an eigenvalue by the Riesz theory. To conclude the proof we must show that if $\lambda$ is in the spectrum of $T_{L}$ and $\left(\lambda I-T_{L}\right)^{-1}$ exists, then zero is in the spectrum of $S-\hat{L}-P(\lambda)$. Clearly, $\mathscr{R}\left(T_{L}-\lambda I\right)$ cannot be dense, because it is closed. But then $\bar{\lambda}$ is an eigenvalue of $T_{L *}$. It follows that zero is an eigenvalue of $S-\hat{L}^{*}-P(\bar{\lambda})$, and hence of $S-\hat{L}-P(\lambda)$.

To determine values of $\lambda$ such that $0 \in \rho(S-\hat{L}-P(\lambda))$ we shall need two lemmas. The first of these follows directly from the argument of Lax and Milgram [17], p. 169.

5.12. Lemma. Let $V$ be a densely defined, closed, linear operator in Hilbert space such that $\mathscr{D}(V)=\mathscr{D}\left(V^{*}\right)$. Suppose there is a constant $K>0$ for which we have

$$
|(V u, u)| \geqq K\|u\|^{2}, \quad u \in \mathscr{D}(V) .
$$

Then zero is in the resolvent set of $V$, and $\left\|V^{-1}\right\| \leqq K^{-1}$.

The next lemma may be found in Ehrling [10], p. 270.

5.13. Lemma. There exist constants $M \geqq 1$ and $t_{0} \geqq 1$ such that

$$
\|\tilde{u}\|^{2} \leqq M t^{-1 / 2}\left(D(u, u)+t\|u\|^{2}\right), \quad t \geqq t_{0}, \quad u \in W^{1,2}(G) .
$$

In applying these lemmas, we determine conditions on $\lambda$ such that

$$
|((S-P(\lambda))[u, \tilde{u}],[u, \tilde{u}])|>\|L\|\|[u, \tilde{u}]\|^{2}, \quad u \in \mathscr{D}_{1}(G) .
$$

For such $\lambda$, we have $0 \in \rho(S-\hat{L}-P(\lambda))$, because $\left\|(S-P(\lambda))^{-1}\right\|<\|L\|^{-1}$ by Lemma 5.12, and

$$
(S-\hat{L}-P(\lambda))^{-1}=[S-P(\lambda)]^{-1} \sum_{n=0}^{\infty}(-1)^{n}\left[\hat{L}(S-P(\lambda))^{-1}\right]^{n},
$$

the series converging in the uniform operator topology. Let $s$ be a parameter, $0<s<1$. Writing $\lambda=\sigma+i \tau$, we have, by Green's identity and Lemma 5.13,

$$
\begin{aligned}
|((S-P(\lambda))[u, \tilde{u}],[u, \tilde{u}])| & =\left|D(u, u)-\lambda\|u\|^{2}\right| \\
& \geqq s D(u, u)+[(1-s)|\tau|-s \sigma]\|u\|^{2} \\
& =s\left[D(u, u)+\frac{[(1-s)|\tau|-s \sigma]\|u\|^{2}}{2 s}\right] \\
& +\frac{[(1-s)|\tau|-s \sigma]\|u\|^{2}}{2} \\
& \geqq \frac{s^{1 / 2}[(1-s)|\tau|-s \sigma]^{1 / 2}\|[u, \tilde{u}]\|^{2}}{2 M},
\end{aligned}
$$

if $(1-s)|\tau|-s \sigma>2 t_{0}$. Suppose, in addition, that 


$$
(1-s)|\tau|-s \sigma>C s^{-1},
$$

where $C$ is the larger of $2 t_{0}$ and $4 M^{2}\|L\|^{2}$. Then (*) is satisfied, and therefore $\lambda$ is in the resolvent set of $T_{L}$, by Lemma 5.11. Rewriting $\left({ }^{* *}\right)$ in the form

$$
|\tau|>\frac{s \sigma}{1-s}+\frac{C}{s(1-s)},
$$

we see $\sigma\left(T_{\Sigma}\right)$ lies in each of the sectors

$$
\mu=-C s^{-2}+r e^{i \phi},|\tan \phi| \leqq \frac{s}{1-s}, \quad r \geqq 0 .
$$

For each fixed value of $\sigma>0$, we may compute the minimum value of $\tau$ satisfying

$$
\tau=\frac{s \sigma}{1-s}+\frac{C}{s(1-s)}
$$

for $0<s<1$. The result is a complicated expression which is $O\left(\sigma^{1 / 2}\right)$ as $\sigma \rightarrow+\infty$. We summarize the situation in

5.14. THEOREM. Let $L$ be a bounded operator in $L_{2}(\partial G)$. The operator $T_{L}$ with domain

$$
\mathscr{D}\left(T_{L}\right)=\left\{u \in \mathscr{D}_{1}(G) \mid \widetilde{u}_{n}=L \tilde{u}\right\}
$$

and

$$
T_{L} u=-\Delta u, \quad u \in \mathscr{D}\left(T_{L}\right),
$$

is a closed linear operator with compact resolvent, whose spectrum lies inside a parabola $\tau^{2}=a \sigma+b, a, b>0$. Moreover, $T_{L}{ }^{*}=T_{L *}$.

All has been proved except the compactness of the resolvent. It is enough to prove it for one nonzero value of $\lambda$. If $\left\{\left(\lambda I-T_{L}\right) u^{k}\right\}$ is a bounded sequence, the sequences $\left\{u^{k}\right\}$ and $\left\{\Delta u^{k}\right\}$ are bounded (since $\left(\lambda I-T_{L}\right)^{-1}$ is bounded). Thus $\left\{\widetilde{u}_{k}\right\}$ is bounded, by Corollary 5.7. It follows from Green's identity that $\left\{u^{k}\right\}$ is bounded in $W^{1,2}(G)$, and therefore a sub-sequence of $\left\{u^{k}\right\}$ converges in $L_{2}(G)$ (cf. the proof of 4.2).

\section{REFERENCES}

1. N. Aronszajn and K. T. Smith, Functional spaces and functional completion, Ann. Inst. Fourier, Grenoble, 6 (1955-56), 125-185.

2. F. E. Browder, On the regularity properties of solutions of elliptic differential equations, Comm. on Pure and Applied Math., 9 (1956), 351-361.

3. - On the spectral theory of strongly elliptic differential operators, Proc. Nat. Acad. Sci., 45 (1959), 1423-1431. 
4. A. P. Calderon and A. Zygmund, On the existence of certain singular integrals, Acta Math., 88 (1952), 85-139.

5. J. W. Calkin, Abstract self-adjoint boundary conditions, Proc. Nat. Acad. Sci., 24 (1938), $38-42$.

6. - Abstract symmetric boundary conditions, Trans. Amer. Math. Soc., 45 (1939), $369-442$.

7. - General self-adjoint boundary conditions for certain partial differential operators, Proc. Nat. Acad. Sci., 25 (1939), 201-206.

8. - Abstract definite boundary value problems, ibid., 26 (1940), 708-712.

5. N. Dunford and J. T. Schwartz, Linear Operators, Part I: General theory, New York, 1958.

10. G. Ehrling, On a type of eigenvalue problem for certain elliptic differential operators, Math. Scand., 2 (1954) 267-285.

11. P. Frank and R. von Mises, Die Differential and Integralgleichungen der Mechanik und Physik, vol. 1, Braunschweig, 1930.

12. R. S. Freeman, Self-adjoint boundary conditions for the Laplace operator, Thesis, University of California, Berkeley, 1959.

13. Closed extensions of the Laplace operator determined by a general class of boundary conditions for unbounded regions, to appear.

14. K. O. Friedrichs, Spektraltheorie halbbeschränkter Operatoren und Anwendung auf die Spektralzerlegung von Differentialoperatoren I, II, Math. Ann., 109 (1934), 465-486, 685-713.

15. J. T. Joichi, On closed operators with closed range, Proc. Amer. Math. Soc., 11 (1960), $80-83$.

16. O. D. Kellogg, Foundations of potential theory, Springer, Berlin, 1929.

17. P. Lax and A. Milgram, Parabolic equations, Ann. of Math. Studies No. 33, Princeton, 1954.

18. K. R. Lucas, Submanifolds of dimension $n-1$ in $E^{n}$ with normals satisfying a Lipschitz condition, Studies in eigenvalue problems, Technical Report 18, University of Kansas, 1957.

19. C. B. Morrey, Jr., Functions of several variables and absolute continuity II, Duke Math. J., 6 (1940), 186-215.

20. J. Odhnoff, Operators generated by differential problems with eigenvalue parameter in equation and boundary condition, Thesis, Lund University, 1959.

21. J. W. Smith, Two classes of self-adjoint boundary conditions for the Laplacian operator, Thesis, University of California, Berkeley, 1950.

22. W. J. Sternberg and T. L. Smith, The theory of potential and spherical harmonics, Toronto, 1944.

UNIVERSITY OF CALIFORNIA, BERKELEY

LAWRENCE RADIATION LABORATORY, UNIVERSITY OF CALIFORNIA

LIVERMORE, CALIFORNIA 


\title{
PACIFIC JOURNAL OF MATHEMATICS
}

\author{
EDITORS
}

Ralph S. Phillips

Stanford University

Stanford, California

M. G. Arsove

University of Washington

Seattle 5, Washington
A. L. Whiteman

University of Southern California Los Angeles 7, California

Lowell J. Paige

University of California

Los Angeles 24, California

\section{ASSOCIATE EDITORS}
E. F. BECKENBACH
D. DERRY
M. OHTSUKA
H. L. ROYDEN
E. SPANIER
E. G. STRAUS
T. M. CHERRY
F. WOLF

\section{SUPPORTING INSTITUTIONS}

\author{
UNIVERSITY OF BRITISH COLUMBIA \\ CALIFORNIA INSTITUTE OF TECHNOLOGY \\ UNIVERSITY OF CALIFORNIA \\ MONTANA STATE UNIVERSITY \\ UNIVERSITY OF NEVADA \\ NEW MEXICO STATE UNIVERSITY \\ OREGON STATE UNIVERSITY \\ UNIVERSITY OF OREGON \\ OSAKA UNIVERSITY \\ UNIVERSITY OF SOUTHERN CALIFORNIA
}

\author{
STANFORD UNIVERSITY \\ UNIVERSITY OF TOKYO \\ UNIVERSITY OF UTAH \\ WASHINGTON STATE UNIVERSITY \\ UNIVERSITY OF WASHINGTON \\ $*$
AMERICAN MATHEMATICAL SOCIETY \\ CALIFORNIA RESEARCH CORPORATION \\ SPACE TECHNOLOGY LABORATORIES \\ NAVAL ORDNANCE TEST STATION
}

Mathematical papers intended for publication in the Pacific Journal of Mathematics should be typewritten (double spaced), and the author should keep a complete copy. Manuscripts may be sent to any one of the four editors. All other communications to the editors should be addressed to the managing editor, L. J. Paige at the University of California, Los Angeles 24, California.

50 reprints per author of each article are furnished free of charge; additional copies may be obtained at cost in multiples of 50 .

The Pacific Journal of Mathematics is published quarterly, in March, June, September, and December. Effective with Volume 13 the price per volume (4 numbers) is $\$ 18.00$; single issues, $\$ 5.00$. Special price for current issues to individual faculty members of supporting institutions and to individual members of the American Mathematical Society: $\$ 8.00$ per volume; single issues $\$ 2.50$. Back numbers are available.

Subscriptions, orders for back numbers, and changes of address should be sent to Pacific Journal of Mathematics, 103 Highland Boulevard, Berkeley 8, California.

Printed at Kokusai Bunken Insatsusha (International Academic Printing Co., Ltd.), No. 6, 2-chome, Fujimi-cho, Chiyoda-ku, Tokyo, Japan.

PUBLISHED BY PACIFIC JOURNAL OF MATHEMATICS, A NON-PROFIT CORPORATION

The Supporting Institutions listed above contribute to the cost of publication of this Journal, but they are not owners or publishers and have no responsibility for its content or policies. 


\section{Pacific Journal of Mathematics}

\section{Vol. 12, No. $2 \quad$ February, 1962}

William George Bade and Robert S. Freeman, Closed extensions of the Laplace operator determined by a general class of boundary conditions . . . . . . . 395

William Browder and Edwin Spanier, H-spaces and duality ............. 411

Stewart S. Cairns, On permutations induced by linear value functions . . . . . . . 415

Frank Sydney Cater, On Hilbert space operators and operator roots of

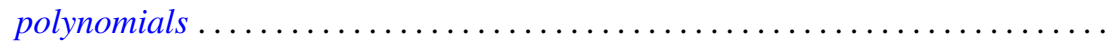

Stephen Urban Chase, Torsion-free modules over $K[x, y] \ldots \ldots \ldots \ldots \ldots \ldots \ldots 437$

Heron S. Collins, Remarks on affine semigroups . . . . . . . . . . . . . . . . 449

Peter Crawley, Direct decompositions with finite dimensional factors . . . . . . . 457

Richard Brian Darst, A continuity property for vector valued measurable

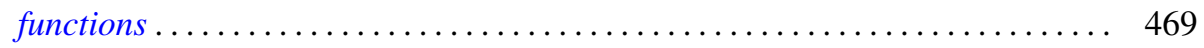

R. P. Dilworth, Abstract commutative ideal theory ................. 481

P. H. Doyle, III and John Gilbert Hocking, Continuously invertible spaces . . . . . . 499

Shaul Foguel, Markov processes with stationary measure . . . . . . . . . . . 505

Andrew Mattei Gleason, The abstract theorem of Cauchy-Weil ............ 511

Allan Brasted Gray, Jr., Normal subgroups of monomial groups . . . . . . . . . . 527

Melvin Henriksen and John Rolfe Isbell, Lattice-ordered rings and function

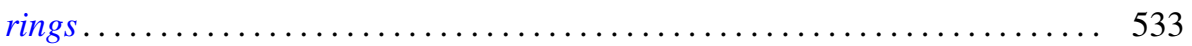

Amnon Jakimovski, Tauberian constants for the $[J, f(x)]$ transformations . ..... 567

Hubert Collings Kennedy, Group membership in semigroups . . . . . . . . . . . 577

Eleanor Killam, The spectrum and the radical in locally $m$-convex algebras ..... 581

Arthur H. Kruse, Completion of mathematical systems . . . . . . . . . . . . . 589

Magnus Lindberg, On two Tauberian remainder theorems ................ 607

Lionello A. Lombardi, A general solution of Tonelli's problem of the calculus of

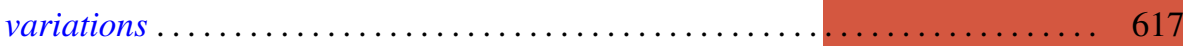

Marvin David Marcus and Morris Newman, The sum of the elements of the powers

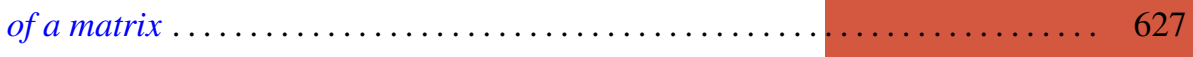

Michael Bahir Maschler, Derivatives of the harmonic measures in

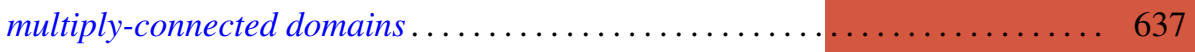

Deane Montgomery and Hans Samelson, On the action of $\mathrm{SO}(3)$ on $S^{n} \ldots \ldots \ldots 649$

J. Barros-Neto, Analytic composition kernels on Lie groups . . . . . . . . . . . . 661

Mario Petrich, Semicharacters of the Cartesian product of two semigroups ...... 679

John Sydney Pym, Idempotent measures on semigroups . . . . . . . . . . . . 685

K. Rogers and Ernst Gabor Straus, A special class of matrices . . . . . . . . . . . . 699

U. Shukla, On the projective cover of a module and related results . . . . . . . . . 709

Don Harrell Tucker, An existence theorem for a Goursat problem . . . . . . . . . . . 719

George Gustave Weill, Reproducing kernels and orthogonal kernels for analytic

differentials on Riemann surfaces ......................... 729

George Gustave Weill, Capacity differentials on open Riemann surfaces ........ 769

G. K. White, Iterations of generalized Euler functions . . . . . . . . . . . . . 777

Adil Mohamed Yaqub, On certain finite rings and ring-logics . . . . . . . . . 785 